\title{
MICROESTRUCTURA Y RESISTENCIA A LA CORROSIÓN DE NITRUROS DE CROMO OBTENIDOS POR NITRURACIÓN GASEOSA AL VACÍO DE CROMO ELECTROLIITICO DEPOSITADO SOBRE ACERO AISI HI 3
}

\author{
H. Cifuentes \\ J. J. Olaya ${ }^{* *}$
}

Recibido: 26/01/2012

Aceptado: 07/05/2013

\section{RESUMEN}

En este artículo se caracteriza la microestructura y se evalúa la resistencia a la corrosión de nitruros de cromo obtenidos a partir de un tratamiento dúplex constituido por un recubrimiento electrolítico de cromo aplicado sobre un acero AISI H13 combinado posteriormente a un proceso de nitruración gaseosa, mediante un tratamiento termoquímico al vacío, utilizando $\mathrm{NH}_{3}$ como gas precursor de nitrógeno. Este tipo de tratamientos dúplex permite combinar las ventajas de tratamientos individuales y con este efecto sinérgico, obtener compuestos tipo $\mathrm{Cr}_{\mathrm{x}} \mathrm{N}$ en forma más económica que los producidos tradicionalmente de deposición física en fase de vapor (PVD). Los resultados obtenidos mediante difracción de rayos-X (XRD) indican una transformación superficial y subsuperficial del recubrimiento electrolítico de cromo con formación de fases $\mathrm{CrN}$ y $\mathrm{Cr}_{2} \mathrm{~N}$. Así mismo, los ensayos de polarización potenciodinámica indican un incremento en la resistencia a la corrosión de este tipo de compuestos en comparación con los obtenidos con cromo duro electrolítico.

Palabras clave: Nitruros de cromo, tratamientos dúplex, tratamientos termoquímicos al vacío, resistencia a la corrosión, ensayos de polarización potenciodinámica.

Ingeniero Mecánico MsC en Educación, Universidad Pedagógica Nacional. Doctor en Ingeniería - Ciencia y Tecnología de Materiales, Universidad Nacional de Colombia. Bogotá, Colombia. Universidad Nacional de Colombia, Sede Bogotá. E-mail: hcifuentesa@unal.edu.co

Ingeniero Metalúrgico. M.Sc., en Materiales y Procesos, Universidad Nacional de Colombia. Ph.D., en Ingeniería, Universidad Nacional Autónoma de México. Universidad Nacional de Colombia Sede Bogotá. E-mail: jjolayaf@unal.edu.co 


\title{
MICROSTRUCTURE AND CORROSION RESISTANCE OF CHROMIUM NITRIDES OBTAINED BY VACUUM GAS NITRIDING OF ELECTROLYTIC CHROMIUM DEPOSITED ON AISI HI 3 STEEL
}

\begin{abstract}
In this scientific research paper, the microstructure and corrosion resistance of chromium nitrides obtained from a duplex treatment consisting of an electroplated hard chromium coating applied on a steel AISI H13 follow by a thermochemical treatment in vacuum using $\mathrm{NH} 3$ as precursor gas of nitrogen, is evaluated. This type of duplex treatments combine the benefits of each individual treatment in order to obtain, with this synergic effect, compounds type CrxN more economic than those obtained by other kind of treatments e.g. physical vapor deposition (PVD). The results obtained by X-Ray Diffraction (XRD) indicate the surface and subsurface transformation of the electrolytic hard chromium coating by formation of $\mathrm{CrN}$ and $\mathrm{Cr} 2 \mathrm{~N}$ phases. Likewise, potentiodynamic polarization tests indicate an increase in corrosion resistance of such kind of compounds in comparison with the obtained results with electroplated hard chromium.
\end{abstract}

Key words: Chromium nitrides, duplex treatments, vacuum thermochemical treatments, corrosion resistance, potenciodinamyc polarization tests. 


\section{INTRODUCCIÓN}

El desarrollo de los materiales ha sido paralelo a la evolución de las civilizaciones, y el ser humano los ha utilizado para la fabricación de diverso tipo de elementos (instrumentos, equipos, máquinas) que le han permitido mejorar su nivel de vida y facilitar tareas que sin su utilización no hubieran sido posibles. En la actualidad constituyen una parte fundamental de la economía y tanto científicos como ingenieros realizan investigaciones orientadas ya sea a la producción de nuevos materiales, como a la modificación de los ya existentes con el objetivo de mejorar sus propiedades. Esta búsqueda obedece a que, en general, los elementos constitutivos de máquinas, equipos o instrumentos se encuentran sometidos a diferentes tipos de fenómenos físicos y/o químicos que pueden conducir a la pérdida gradual de su integridad funcional y del rendimiento del sistema de ingeniería al cual pertenecen como resultado de procesos de degradación como corrosión, desgaste, fatiga, etc. Debido a que estos fenómenos inician generalmente en la superficie del material o sustrato se ha desarrollado un conjunto de tratamientos con el objetivo de modificarlos superficial y/o subsuperficialmente y generar una interface con propiedades específicas que permitan al sistema metalúrgico soportar las solicitaciones $\mathrm{y} / \mathrm{o}$ exigencias funcionales y del entorno, y cumplir funciones diferentes a las requeridas por el sustrato [1] manteniéndolo, a su vez, dentro de los parámetros de ingeniería exigidos.

Esta interface se puede obtener mediante métodos que involucran el crecimiento intencional o la adición de una nueva capa (por ejemplo, plateado electroquímico) o con métodos que involucran la modificación de la superficie sin crecimiento intencional o incremento en dimensiones de la pieza (por ejemplo, procesos difusivos). El proceso de recubrimiento electrolítico de cromo duro hexavalente $\left(\mathrm{Cr}_{(\mathrm{VI})}\right)$ sobre sustratos de acero es un ejemplo del primer conjunto de métodos y es una técnica ampliamente utilizada como método de protección y mejora de características superficiales de diversos elementos de máquinas, herramientas, componentes automotrices, aeronáuticos y de la industria en general [2-6], por el incremento que producen en la magnitud de propiedades como resistencia a la corrosión, resistencia al desgaste y dureza, así como la disminución en valores de coeficientes de fricción. Sin embargo, está técnica presenta una serie de inconvenientes, como por ejemplo, la generación de microgrietas producto del desarrollo y relevo de esfuerzos residuales en el sistema sustrato/recubrimiento durante el proceso de deposición del $\mathrm{Cr}_{(\mathrm{VII})}$ [7]. Estas microgrietas tienen efectos adversos sobre la superficie ya que, entre otros aspectos, afectan las propiedades antes mencionadas, en especial, a temperaturas superiores a $623 \mathrm{~K}$ [2] debido a la facilidad con que los agentes oxidantes difunden y alcanzan el sustrato a través de estos defectos [2, 5]. De otra parte, diferentes reportes y estudios muestran que el $\mathrm{Cr}_{(\mathrm{VI})}$ es altamente contaminante y lesivo para la salud humana ya que se le considera posible fuente de enfermedades cancerígenas [8].

La obtención de sistemas basados en materiales cerámicos, tales como nitruros de cromo $\mathrm{Cr}_{\mathrm{x}} \mathrm{N}$ es una alternativa de protección para materiales utilizados en instalaciones industriales bajo condiciones ambientales severas (por ejemplo, corrosión salina) ya que su presencia incrementa la vida útil de los componentes y sistemas en que se integran al mejorar características funcionales de las superficies de aceros como sus propiedades mecánicas, resistencia a la corrosión $[2,3,9,10]$, resistencia al desgaste $[2,4,11,12]$, o disminuyen la resistencia eléctrica al contacto interfacial en aplicaciones como celdas de combustible [9]. Este tipo de compuestos $\left(\mathrm{Cr}_{\mathrm{x}} \mathrm{N}\right)$ exhiben ventajas comparativas respecto a otros compuestos cerámicos como el nitruro de titanio ya que permiten obtener mayores valores de dureza [11] o mejor resistencia al desgaste y la corrosión [12].

Se han desarrollado diferentes técnicas para la obtención de películas tipo $\mathrm{Cr}_{\mathrm{x}} \mathrm{N}$ sobre sustratos ferrosos como por ejemplo, la deposición directa 
a partir de deposición física en fase vapor (PVD, por sus siglas en inglés). Sin embargo, esta técnica se ve limitada por aspectos como su alto costo de producción y su mayor control en los parámetros de depósito [13], bajos espesores (alrededor de 5 $\mu \mathrm{m})$ y altos esfuerzos residuales de compresión generados por la elevada diferencia existente entre los coeficientes de expansión térmica de los compuestos $\mathrm{Cr}_{\mathrm{x}} \mathrm{N}$ y el sustrato de acero (relación 1:6) especialmente en aplicaciones a alta temperatura $[5,12]$.

Una alternativa económica que permite obtener recubrimientos cerámicos duros y en particular nitruros de cromo $\left(\mathrm{Cr}_{\mathrm{x}} \mathrm{N}\right)$, se ha logrado a partir de la realización de tratamientos superficiales combinados o dúplex [14]. Estos permiten transformar, por ejemplo, recubrimientos electrolíticos de cromo duro hexavalente hacia compuestos cerámicos tipo $\mathrm{Cr}_{\mathrm{x}} \mathrm{N}$ generando un impacto positivo en aspectos medioambientales y de salud pública, ya que al incrementar la vida útil del recubrimiento, se disminuye el consumo de $\mathrm{Cr}_{(\mathrm{VI})}$ a escala industrial. Esta transformación hacia cerámicos tipo $\mathrm{Cr}_{\mathrm{x}} \mathrm{N}$ permite, además, eliminar defectos como las microgrietas producidas durante el proceso electroquímico. De otra parte, su estabilidad a alta temperatura los hace apropiados para aplicaciones en moldes, herramientas de corte y componentes de soporte. En particular, se han desarrollado procesos dúplex que combinan un proceso electroquímico para la obtención de recubrimientos de cromo duro sobre un sustrato ferroso, con tratamientos termoquímicos posteriores de nitruración mediante técnicas con plasma [2, 10] o en atmósfera gaseosa [3, 5, 9, 15].

El objetivo de este trabajo es producir recubrimientos tipo $\mathrm{Cr}_{\mathrm{X}} \mathrm{N}$ mediante un proceso dúplex constituido inicialmente por un proceso electroquímico con el cual se recubre con cromo duro la superficie de un acero AISI H13, seguido de un tratamiento termoquímico en atmósfera gaseosa al vacío que permite, mediante la adición de gases precursores de nitrógeno como amoniaco
$\left(\mathrm{NH}_{3}\right)$ o nitrógeno $\left(\mathrm{N}_{2}\right)$, transformar esta superficie hacia nitruros de cromo tipo $\mathrm{Cr}_{\mathrm{x}} \mathrm{N}$ y correlacionar la microestructura y la resistencia a la corrosión en los recubrimientos producidos. El tratamiento termoquímico es un proceso de tipo heterogéneo y se desarrolla entre una fase fluida (gas) y un sólido (superficie de un sustrato). La obtención de especies atómicas de interés requeridas para el proceso se logra a partir del desarrollo de una serie de fenómenos con base en parámetros establecidos de composición química, presión, temperatura, flujo y tiempo $[16,17]$. Un aspecto fundamental para el adecuado desarrollo de tratamientos termoquímicos en atmósfera gaseosa es el control de su composición ya que está constituida primero, por los gases que permitirán la obtención de las especies atómicas requeridas con las cuales se desarrollarán las fases y microestructuras específicas en la superficie del sustrato y segundo, por gases que por su presencia pueden deteriorar el proceso al generar reacciones no deseadas (por ejemplo, oxidación). Una técnica que permite este control es el vacío que, además, suministra una adecuada protección atmosférica minimizando el riesgo de posibles reacciones de oxidación o descarburación superficial, un control preciso de variables como temperatura, tiempo y/o presión indispensable para la repetibilidad del tratamiento, ausencia de daño superficial relacionado con el contacto que se produce cuando se aplican otros tratamientos que involucran sólidos o sales, fácil mantenimiento y reducción de tiempo de proceso en $60 \%$ por incremento en velocidades de calentamiento y disminución de costos de operación [6].

\section{DESARROLLO EXPERIMENTAL}

Los recubrimientos tipo $\mathrm{Cr}_{\mathrm{x}} \mathrm{N}$ se produjeron mediante un tratamiento dúplex constituido por un recubrimiento electrolítico de cromo duro $\left(\mathrm{Cr}_{(\mathrm{VI})}\right)$, con un tratamiento termoquímico posterior de nitruración gaseosa realizado en un horno eléctrico con cámara al vacío. Como sustrato se utilizaron discos de 25,4 mm de diámetro y espesor de 1,5 \pm

Universidad de Medellín 
$0,2 \mathrm{~mm}$, rectificados y pulidos con lija hasta grado 600 y fabricados en acero AISI H13 con composición química en porcentaje en peso de C: 0,40 \%; Si: 1,00 \%; Cr: 5,20 \%; Mo: 1,30 \%; V: 0,95 \%.

\subsection{Recubrimiento electrolítico}

Los recubrimientos de $\mathrm{Cr}_{(\mathrm{VI})}$ duro aplicados sobre el acero AISI H13 se produjeron por vía electroquímica en un baño electrolítico según la composición mostrada en la tabla 1 , a una temperatura de $40^{\circ} \mathrm{C}$, con densidad de corriente catódica de $30 \mathrm{~A} / \mathrm{dm}^{2}$ y duración de $80 \mathrm{~min}$. El espesor de recubrimiento fue de aproximadamente $15 \mu \mathrm{m}$ y se seleccionó con base en criterios de utilización para aplicaciones en manufactura relacionadas con el acero seleccionado (AISI H13).

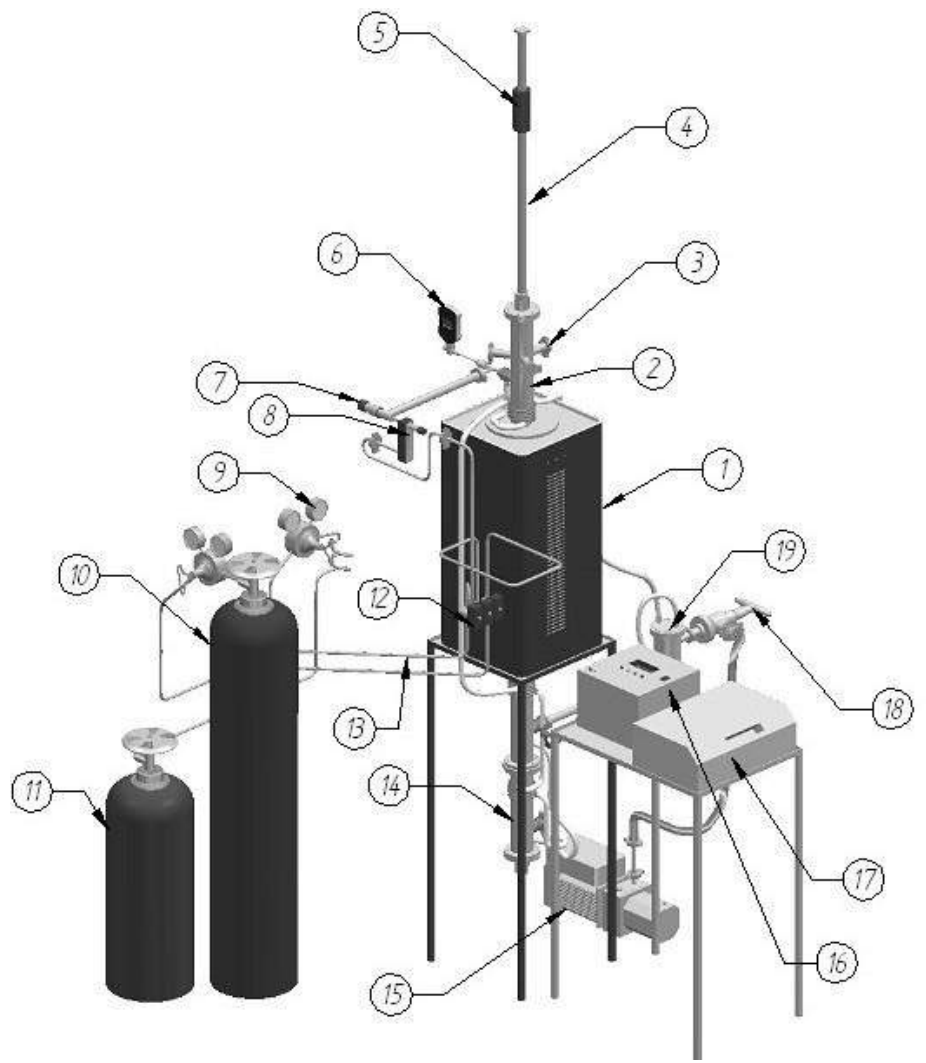

\subsection{Tratamiento termoquímico}

Para la realización del tratamiento dúplex, se construyó un horno con cámara de vacío para tratamientos en atmósfera gaseosa controlada, como el mostrado en la figura 1 . Su diseño permite realizar tratamientos termoquímicos de nitruración, car-

Tabla 1. Composición química

baño de cromo duro electrolítico

\begin{tabular}{|l|l|l|}
\hline \multicolumn{1}{|c|}{ Característica } & \multicolumn{1}{c|}{ Valor } & Unidad \\
\hline Densidad & 24 & oBe \\
\hline Cromo total & 280 & $\mathrm{~g} / 1$ \\
\hline $\mathrm{Cr}+6$ & $250-275$ & $\mathrm{~g} / 1$ \\
\hline $\mathrm{Cr}+3$ & $0-5$ & $\mathrm{~g} / 1$ \\
\hline Sulfatos & $2,5-2,75$ & $\mathrm{~g} / 1$ \\
\hline Fe & 0,5 & $\mathrm{~g} / 1$ \\
\hline Relación Cr+6 vs sulfatos & $100: 1$ & \\
\hline
\end{tabular}

Fuente: Ingecrom Ltda. Proveedor cromo duro

\begin{tabular}{|c|c|c|}
\hline \multicolumn{3}{|c|}{ LISTADO IE PARES } \\
\hline $\begin{array}{c}\text { Numero } \\
\text { del } \\
\text { elemento }\end{array}$ & Titub & Cantidad \\
\hline 1 & Horno & \\
\hline 2 & Precamana y postcamara de Vacio & \\
\hline 3 & Puertos para sondas de analisis de guses & \\
\hline 4 & Manioulador magnetico del portamuestras & \\
\hline 5 & Collarin /mantado & \\
\hline 6 & Medidor de Vacio & \\
\hline 7 & Valvula micrometrica de flujo & \\
\hline 8 & Medidor de flujo & \\
\hline 9 & Valvula reguladora & \\
\hline 10 & Tanque de nitrogeno & \\
\hline 11 & Tanque de acetileno & \\
\hline 12 & Valvula solenoides para gases & \\
\hline 13 & Sistema de alimentacion de gases & \\
\hline 14 & Precamara y postcamana de vacio & \\
\hline 15 & bomba de vacio & \\
\hline 16 & Gabinete de control del horno & \\
\hline 17 & Gabinete de Potencia del horno & \\
\hline 18 & Valvula de cieme & \\
\hline 19 & Trampa de condensados & \\
\hline
\end{tabular}

Figura 1. Horno para tratamientos termoquímicos al vacío

Fuente: Elaboración propia 
burización o nitrocarburización. Está constituido por una cámara de vacío rodeada externamente por un sistema de calentamiento conformado por tres resistencias eléctricas $(220 \mathrm{~V}, 22 \mathrm{~A})$ energizadas desde un tablero de potencia con contactores tipo ON-OFF (17); un sistema de vacío conformado por una bomba mecánica (15) resistente a la corrosión y diseñada específicamente para soportar los efectos corrosivos de gases precursores como el amoniaco $\left(\mathrm{NH}_{3}\right)$, un medidor de vacío (6) con sensor tipo termistor, una trampa de condensados (19), una válvula de aislamiento (18) y una red de tubería en acero inoxidable para evacuar los gases desde la cámara de tratamiento hacia el exterior del sistema. Este sistema permite obtener vacío base hasta $10^{-1}$ Pa y operar con presiones de trabajo hasta atmosférica; un sistema de alimentación de gases conformado por tanques de almacenamiento de $\mathrm{NH}_{3}, \mathrm{C}_{2} \mathrm{H}_{2}$ y $\mathrm{N}_{2}(10,11)$, líneas de alimentación (13) y un conjunto selector de gases conformado por tres válvulas solenoide tipo ON-OFF (12).

En función del tratamiento termoquímico y la especie atómica requerida (nitrógeno o carbono), cada línea permite alimentar un gas precursor diferente (acetileno: línea 1, nitrógeno: línea 2 o amoniaco: línea 3). Para el ajuste del flujo de gases precursores de implementaron un flujómetro (8) y una válvula de aguja micrométrica (7).

El control de variables de proceso se realiza con un sistema de control (17) conformado por un controlador lógico programable (PLC por sus siglas en inglés) y un módulo de adquisición de datos de entrada análoga tipo termocupla, con canales en $\mathrm{mV}, \mathrm{V}$ o mA. El programa de control de proceso se realizó con software Trilogi 6.12.

El equipo cuenta, además, con un sistema de manejo de probetas por manipulador magnético lineal (5) que permite desplazar las muestras dentro de la columna de la cámara de vacío. Dispone de precámara-postcámara de tratamiento para aislar la cámara principal durante montaje y desmontaje de muestras, sin pérdida de vacío.
En la primera parte del tratamiento termoquímico, las probetas cromadas fueron sometidas a limpieza ultrasónica con una fase inicial de desengrase con acetona GR para análisis y un enjuague posterior con 2-propanol GR para análisis (cada fase con duración de 1 minuto). Se realizó vacío hasta un valor de presión base de 2,5 Pa. Simultáneamente, se establecieron en el sistema de control los valores de temperatura, y tiempo de proceso (en minutos), y se seleccionó el gas portador de nitrógeno $\left(\mathrm{NH}_{3}\right)$. Mediante la válvula del flujómetro y la válvula micrométrica de aguja se estableció el valor de flujo de gas que para los diferentes experimentos se mantuvo en $100 \mathrm{ml} / \mathrm{min}$. La presión de trabajo fue $9 \times 10^{2} \mathrm{~Pa}$. Los experimentos se desarrollaron variando la temperatura y el tiempo de tratamiento, de acuerdo con los valores mostrados en la tabla 2.

Tabla 2. Variables de proceso de nitruración

\begin{tabular}{|l|l|}
\hline \multirow{2}{*}{\multicolumn{1}{|c|}{ Variable }} & \multicolumn{1}{|c|}{ Gas de nitruración } \\
\cline { 2 - 2 } & \multicolumn{1}{|c|}{ NH3 } \\
\hline Temperatura $(\mathrm{K})$ & $873,973,1073,1173$ \\
\hline Tiempo $(\mathrm{h})$ & $6,8,10,12$ \\
\hline Flujo $(\mathrm{ml} / \mathrm{min})$ & 100 \\
\hline Presión de trabajo $(\mathrm{Pa})$ & 900 \\
\hline
\end{tabular}

Fuente: Elaboración propia

\subsection{Caracterización estructural y morfológica}

La identificación de las fases presentes y sus orientaciones cristalinas se determinó mediante difracción de Rayos-X (XRD por sus siglas en inglés) con un sistema X-PertPro PAnalytical en modo BraggBrentano con radiación monocromática de $\mathrm{Cu}_{\alpha}$ con longitud de onda $1.540998 \AA$.

La sección transversal y espesor de los recubrimientos tratados se evaluó con microscopía electrónica de barrido (SEM por sus siglas en inglés). Se utilizó un microscopio electrónico de barrido FEI QUANTA 200. La composición química elemental se realizó en el microscopio SEM en modo EDX con un voltaje de $20 \mathrm{kV}$. 


\subsection{Caracterización electroquímica}

Se realizaron mediciones potenciodinámicas en una celda de tres electrodos con electrodo de referencia saturado calumel y contraelectrodo de grafito. El equipo utilizado fue un potenciostato/ galvanostato marca Gamry Instruments referencia 600-06060. La solución utilizada fue preparada con agua previamente destilada y desionizada en un equipo Thermo Scientific modelo D7381. Como soluto se utilizó $\mathrm{NaCl}$ grado analítico en concentración al $3 \%$. Cada probeta se estabilizó
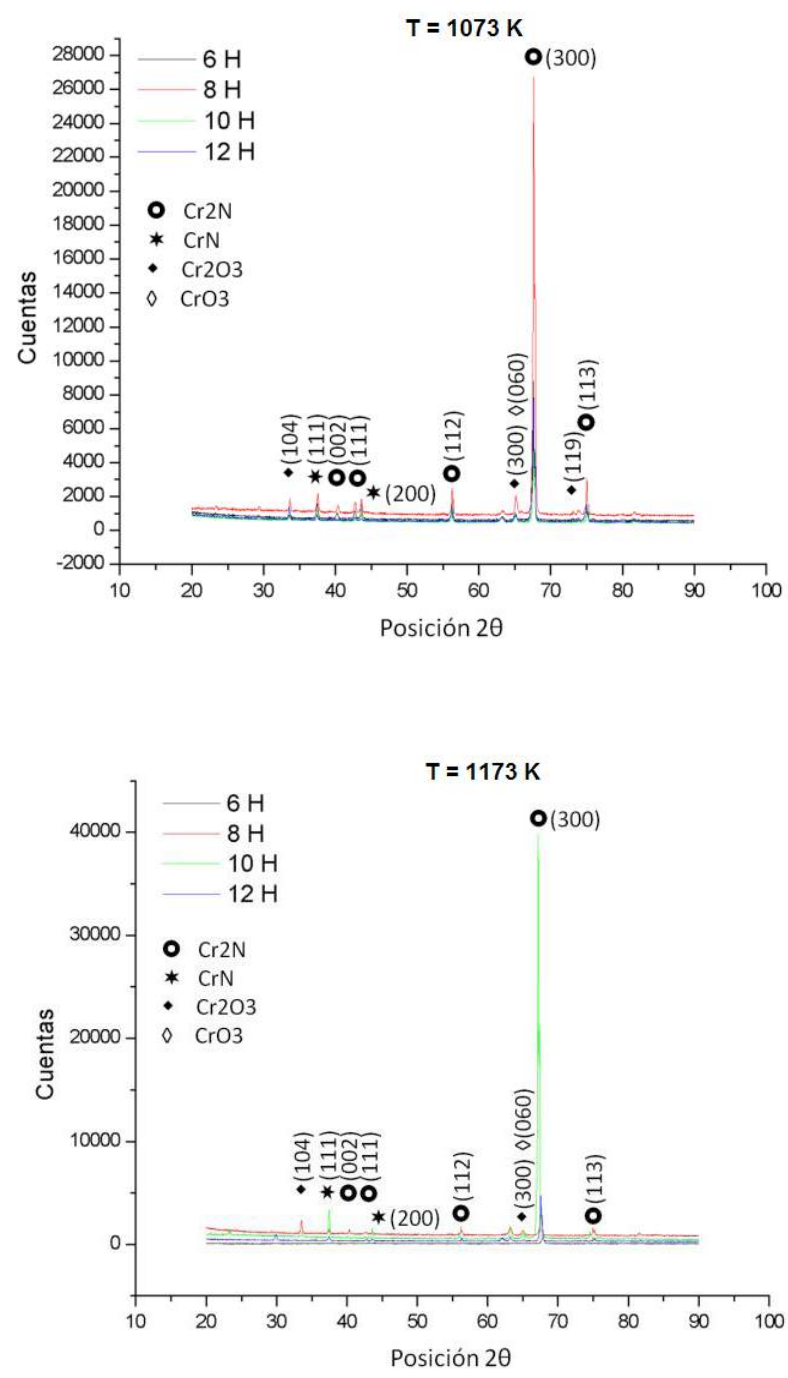

durante 45 minutos antes del inicio de la prueba. Los parámetros para el ensayo de polarización potenciodinámica fueron: voltaje inicial $-0,5 \mathrm{~V}$; voltaje final $0,7 \mathrm{~V}$; tasa de escaneo $0,5 \mathrm{mV} / \mathrm{s}$, periodo de muestreo $1 \mathrm{~s} \mathrm{y}$ área de muestra $0,196 \mathrm{~cm}^{2}$.

\section{RESULTADOSY DISCUSIÓN}

La figura 2 muestra los patrones de difracción obtenidos para el sistema acero H13/cromo electrolítico nitrurado con $\mathrm{NH}_{3}$ en función del tiempo y temperatura de ensayo. Estos patrones se compararon
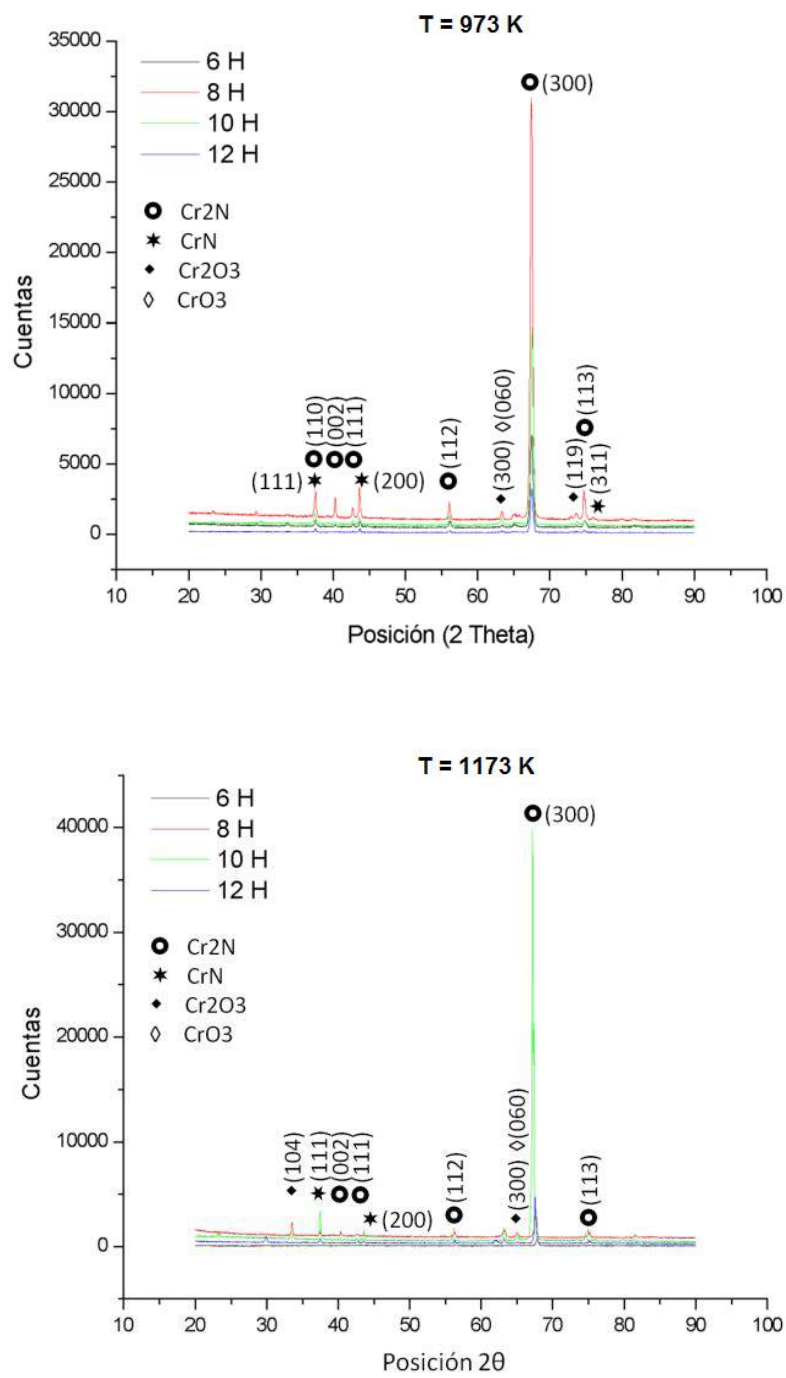

Figura 2. Patrones de difracción de los recubrimientos CrxN en función de tiempo y temperatura de tratamiento Fuente: Elaboración propia 
con los patrones de referencia N. 00-035-0803 para $\mathrm{Cr}_{2} \mathrm{~N}$ y 01-076-2494 para CrN. En general, se observa la formación de la fase cúbica $\mathrm{CrN}$ y de la fase hexagonal $\mathrm{Cr}_{2} \mathrm{~N}$.

Se evidencia la formación de nitruros de cromo tipo CrN en las orientaciones (111) y (200) y tipo $\mathrm{Cr}_{2} \mathrm{~N}$ en las orientaciones (110) (002) (111) (112) (300) (113) y (302) con una elevada orientación preferencial en el plano (300). Este fenómeno de texturización concuerda con resultados reportados en los trabajos de [5] y [9]. En estudios relacionados con mecanismos de crecimiento de películas de nitruro de cromo mediante deposición por arco en vacío con $\mathrm{N}_{2}$ como gas precursor de nitrógeno $[18,19]$ se ha observado esta misma texturización para la fase $\mathrm{Cr}_{2} \mathrm{~N}$ en el plano cristalográfico (300) para una presión de $0,5 \mathrm{~Pa}$ de presión de $\mathrm{N}_{2}$. Los investigadores atribuyen este hecho al contenido de nitrógeno en la película. Concluyen que la estructura y, por tanto, la texturización dependen de las especies precursoras de $\mathrm{N}_{2}$, de sus energías y de la temperatura del sustrato.

Se observa, para las diferentes temperaturas de tratamiento, la formación de un sistema bifásico $\mathrm{CrN}+\mathrm{Cr}_{2} \mathrm{~N}$ lo cual coincide con los trabajos realizados por [12] y [15]. Este hecho obedece a que el incremento de la temperatura favorece la disociación de $\mathrm{NH}_{3}$ y, por tanto, la actividad de

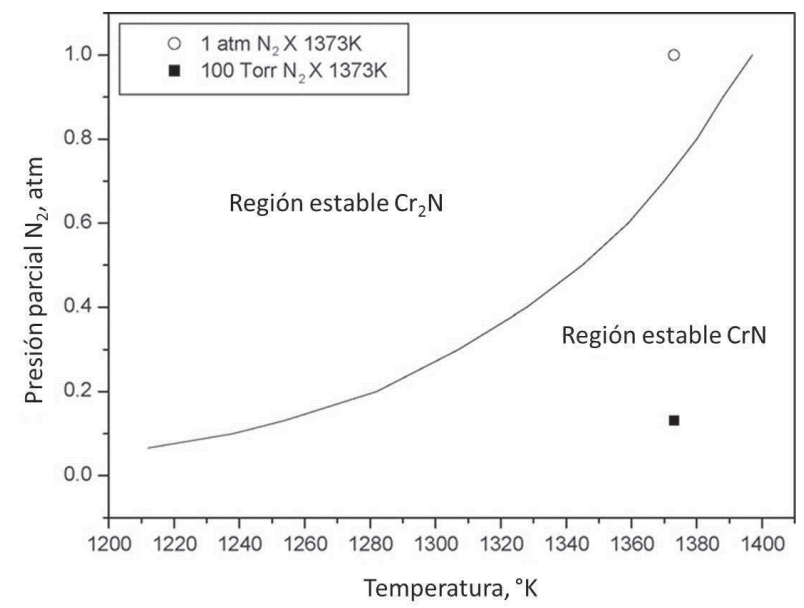

Figura 3. Regiones de estabilidad de nitruros de cromo Fuente: Adaptado de [9] nitrógeno en la atmósfera de tratamiento [15]. La aparición de una u otra fase obedece a la inestabilidad termodinámica que se presenta en función de la temperatura y la presión parcial del gas precursor tal como se ha descrito en [15], donde a partir de cálculos termodinámicos utilizando $\mathrm{N}_{2}$ como gas precursor se obtienen regiones de estabilidad de los nitruros de cromo, como se muestra en la figura 3. Se observa que la formación de fase $\mathrm{Cr}_{2} \mathrm{~N}$ se favorece a menor temperatura de tratamiento y menor presión parcial de gas precursor, como en los tratamientos realizados en este trabajo con temperaturas variables entre $873 \mathrm{~K}$ y $1173 \mathrm{~K}$, con una presión parcial de $1,2 \mathrm{kPa}$.

Se encuentra, además, formación de óxidos de cromo tipo $\mathrm{Cr}_{2} \mathrm{O}_{3}$ y $\mathrm{CrO}_{3}$, en los diferentes tratamientos. Los experimentos se realizaron a bajo vacío (9 Torr) por lo cual existe presencia de oxígeno dentro de la cámara de tratamiento durante la nitruración gaseosa. Sin embargo, estos óxidos (en particular la fase $\mathrm{Cr}_{2} \mathrm{C}_{3}$ ) muestran tendencia a desaparecer con el incremento de la temperatura de tratamiento. Este fenómeno ha sido observado

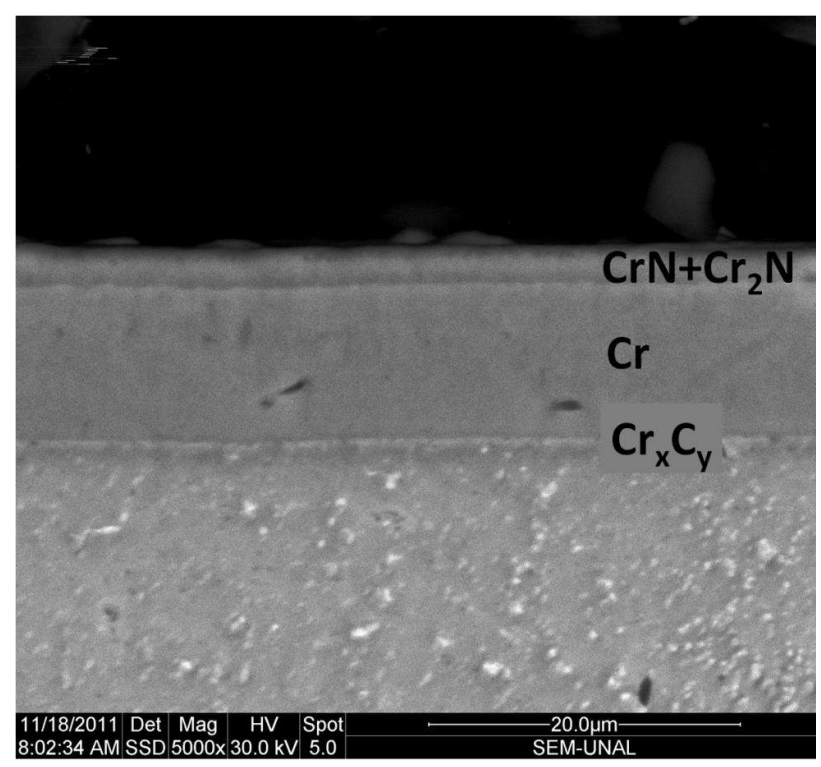

Figura 4. Sección transversal para un tratamiento de nitruración con $\mathrm{NH}_{3}$ a $973 \mathrm{~K}$ y 8 horas

Fuente: Resultados caracterización SEM. Laboratorio CEIFUniversidad Nacional de Colombia - Sede Bogotá. 
en [20] donde se establece una ligera influencia del hidrógeno en esta reducción, elemento que se genera como subproducto de la disociación de amoniaco durante los tratamientos de nitruración realizados. Estas fases fueron comparadas con los patrones de referencia 00-001-1294 para el $\mathrm{Cr}_{2} \mathrm{O}_{3}$ y 00-001-0622 para el $\mathrm{CrO}_{3}$.

El estudio de la sección transversal de los recubrimientos electrolíticos de cromo nitrurados permite observar la transformación de la región del recubrimiento donde se muestra una capa de espesor promedio de $3 \mu \mathrm{m}$ conformada por compuestos cerámicos tipo $\mathrm{Cr}_{\mathrm{x}} \mathrm{N}$ (figura 4) y la formación de en la interface recubrimiento electrolítico de cromo/ acero $\mathrm{H} 13$ de carburos de cromo generados por fenómenos de difusión y reacción de carbono del acero hacia el recubrimiento cromo electrolítico, como los reportados en [2, 3, 15].

En la figura 5 se muestran los resultados obtenidos a partir de los ensayos de polarización

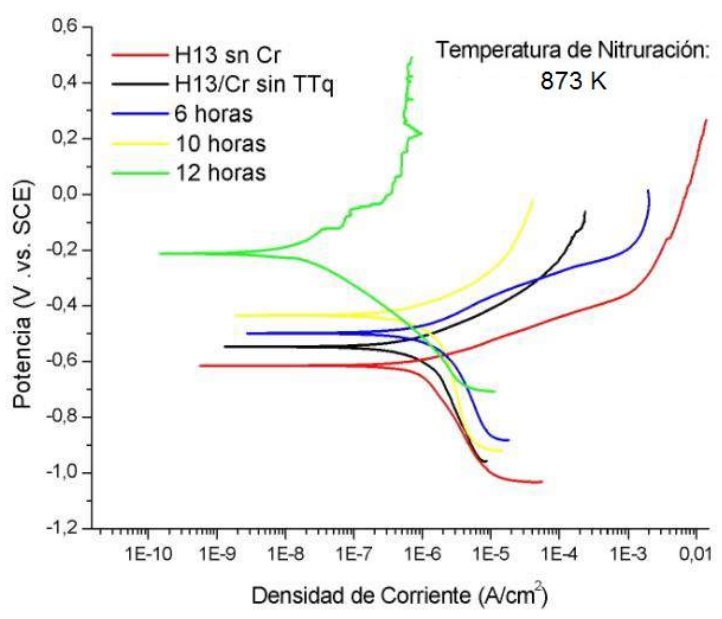

(a)

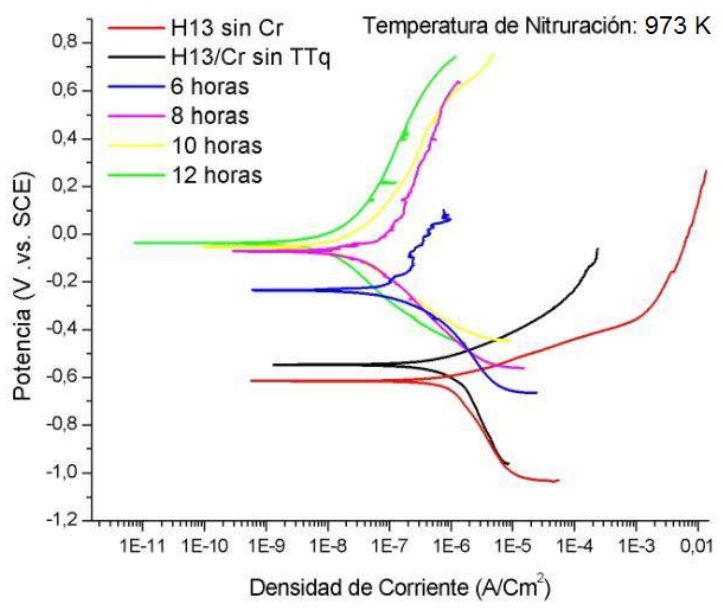

(c)

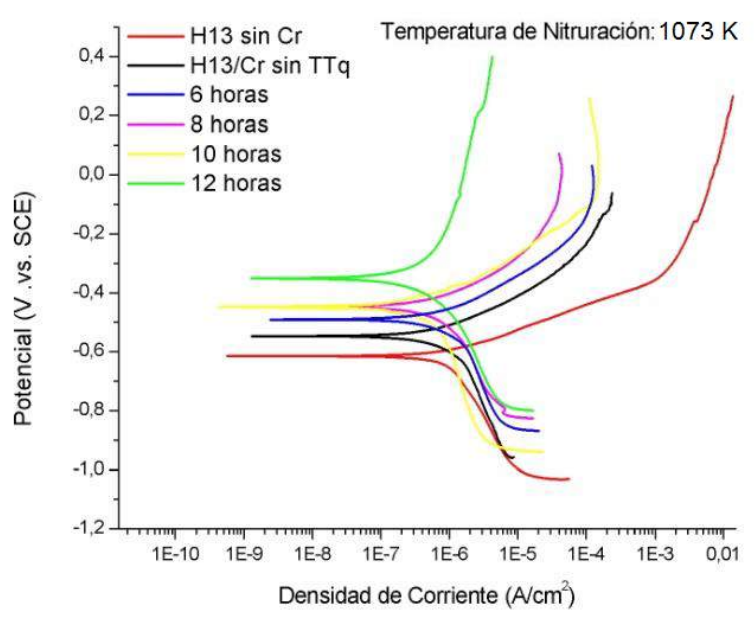

(b)

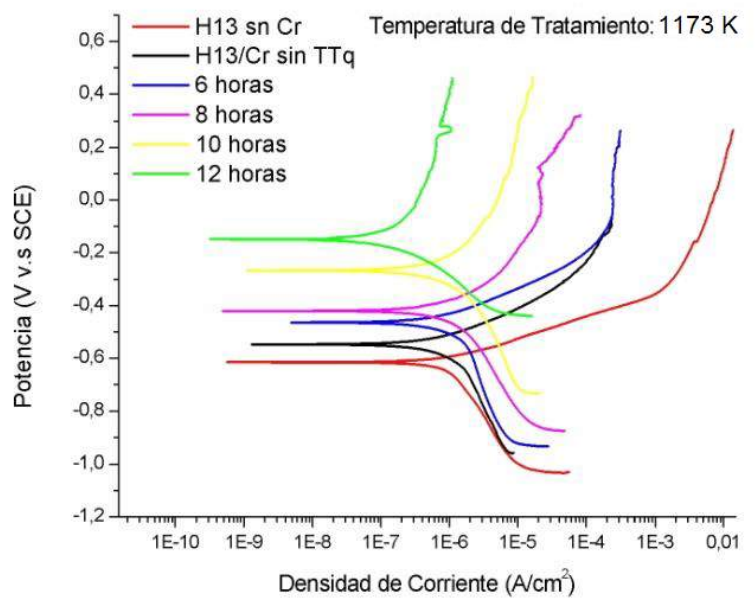

(d)

Figura 5. Curvas de polarización potenciodinámica para acero H13, sistema H13/Cr electrolítico y capas CrxN obtenidas con gas precursor NH3. Temperaturas de tratamiento: (a) $873 \mathrm{~K}$, (b) $973 \mathrm{~K}$, (c) $1073 \mathrm{~K}$, (d) $1173 \mathrm{~K}$

Fuente: Elaboración propia 
potenciodinámica realizados sobre la capa del sistema $\mathrm{Cr} / \mathrm{H} 13$ obtenida con un tratamiento termoquímico de nitruración en atmósfera gaseosa al vacío con amoniaco $\left(\mathrm{NH}_{3}\right)$ y se comparan con el sustrato ferroso (H13) y con el sistema cromado sin nitrurar $(\mathrm{H} 13 / \mathrm{Cr})$.

Los resultados electroquímicos muestran una clara definición de la zona activa-pasiva lo cual permite determinar los parámetros cinéticos para establecer la velocidad de corrosión instantánea de los recubrimientos y el sustrato. Así mismo, se aprecia que el potencial de corrosión de los recubrimientos es más positivo, lo cual indica una mayor resistencia termodinámica al proceso de inicio de la corrosión. Se observa la misma tendencia para la densidad de corriente de corrosión, de manera que las gráficas relacionadas con los recubrimientos se encuentran desplazadas hacia la izquierda. El potencial de corrosión correspondiente al acero H13 sin recubrir tiene un valor de aproximado de $-0,614 \mathrm{~V}$ vs. SCE, mientras que para el recubrimiento electrolítico de cromo es de $-0,546 \mathrm{~V}$ vs. SCE. Este último resultado es coherente con el estudio realizado en [4].

Se observa que los valores de $\mathrm{E}_{\text {corr }}$ en los recubrimientos producidos para temperaturas inferiores y superiores a $973 \mathrm{~K}$ muestran un comportamiento más electropositivo con relación a la $\mathrm{E}_{\text {corr }}$ del cromo sin tratar, y del sustrato ferroso sin recubrir, en particular para tiempos de tratamiento de 6 y 8 horas, con magnitudes de $\mathrm{E}_{\text {corr }}$ alrededor de $-0,469 \mathrm{~V}$. Los tratamientos realizados a $973 \mathrm{~K}$ mostraron una variación significativa de $\mathrm{E}_{\text {corr }} \mathrm{y}$, por lo tanto, un comportamiento más favorable en la electropositividad del sistema, siendo esta superior a mayor tiempo de tratamiento. Esta mejora se observa también para procesos de nitruración a alta temperatura $(1173 \mathrm{~K})$ con tiempos de $10 \mathrm{y}$ 12 horas. Sugiere lo anterior que la presencia de compuestos tipo $\mathrm{Cr}_{\mathrm{x}} \mathrm{N}$ por ser más nobles retrasan los procesos corrosivos.

Se observa que la corriente de corrosión disminuye con el incremento tanto de la temperatura como del tiempo de tratamiento. En las capas producidas a $973 \mathrm{~K}$, se observan los cambios más significativos y se sugiere que la presencia de compuestos tipo $\mathrm{Cr}_{\mathrm{x}} \mathrm{N}$ más compactos, que forman una barrera eficiente que impide que los agentes corrosivos difundan dentro del sistema.

Con base en los resultados del ensayo DRX, se observa que existe mayor presencia de fases CrxN cristalina en los ensayos con temperatura de tratamiento superiores a $973 \mathrm{~K}$. Esto puede confirmar que al aumentar el tiempo y la temperatura de ensayo, se logra aumentar la reactividad del nitrógeno y el cromo, es decir, se aumenta la concentración de $\mathrm{Cr}_{\mathrm{x}} \mathrm{N}$ que mejora la resistencia a la corrosión de todo el sistema.

De otra parte, el mecanismo de corrosión de estos recubrimientos depende de los límites que unen los granos cristalinos, presencia de microporos, pinholes y posibles microgrietas que permiten la difusión de la solución hacia el sustrato y que aceleran el proceso de corrosión localizada (o par galvánico), el cual se favorece para valores de $\mathrm{E}_{\text {corr }}$ del recubrimiento más positivos, comparados con los del acero sin recubrir. Lo anterior produce una diferencia de potencial que permite un ataque acelerado en la interfase recubrimiento-sustrato.

\section{CONCLUSIONES}

La nitruración mediante tratamientos termoquímicos en atmósfera gaseosa en vacío de $\mathrm{Cr}_{(\mathrm{VI})}$ depositado electrolíticamente sobre un sustrato de acero H13 permite la formación con éxito de recubrimientos cerámicos tipo $\mathrm{Cr}_{\mathrm{x}} \mathrm{N}$.

La formación de este tipo de compuestos sugiere un incremento en la resistencia a la corrosión del sistema H13/Cr electrolítico ya que se disminuye la corriente de corrosión; este efecto fue más importante para tratamientos a $973 \mathrm{~K}$ y tiempos superiores a 8 horas de nitruración.

El mecanismo de corrosión en los recubrimientos producidos está basado en la difusión de la solución corrosiva a través de los defectos 
del recubrimiento como grietas y poros, hasta el sustrato. Como consecuencia de esta migración, se crea un par galvánico con disolución del sustrato y pérdida de adherencia en el sistema.

\section{AGRADECIMIENTOS}

Los autores agradecen a la DIB-UNAL por el apoyo económico para realizar esta investigación

\section{REFERENCIAS}

[1] Matthews and A. Leyland, "Hybrid Techniques in Surface Engineering," Surface and Coatings Technology, vol. 71, n. 2 , pp. 88-92, March 1995.

[2] E. Menthe and K-T. Rie, "Plasma Nitriding and Plasma Nitrocarburizing of Electroplated Hard Chromium to Increase the Wear and the Corrosion Properties," Surface and Coatings Technology, vol. 112, n. 1-3, pp. 217-220, February 1999.

[3] D. Kim, M. Kim, K.S. Nam, D. Chang, and S.C. Kwon, "Duplex coating for improvement of corrosion resistance in chromium deposit.," Surface and Coatings Technology, vol. 169-170, n. ${ }^{\circ}$ 2, pp. 650-654, June 2003.

[4] K.-S. Nam, K-H. Lee, S-C. Kwon, D.Y. Lee, and Y-S. Song, "Improved wear and corrosion resistance of chromium (III) plating by oxynitrocarburising and steam oxidation," Materials Letters, vol. 58, n. 27-28, pp. 3540-3544, November 2004.

[5] P. K. Ajikumar et al., "Morphology and Growth Aspects of $\mathrm{Cr}(\mathrm{N})$ Phases on Gas Nitridation of Electroplated Chromium on AISI 316 LN Stainless Steel," Surface and Coatings Technology, vol. 201, n. $1-2$, pp. 102-107, September 2006.

[6] American Society of Materials, ASM Handbook. Heat Treating, Volume 4.: ASM Handbook Committee, 1991.

[7] J. Pina, A. Dias, M. Francois, and J.L. Lebrun, "Residual stresses and crystallographic texture in hard-chromium electroplated coatings," Surface and Costings Technology, vol. 96, n. 2-3, pp. 148-162, November 1997.

[8] Risk and Policy Analysts Ltd., "Enviromental Risk Reduction Strategy and Analysis of Advantages and Drawbacks of Hexavalent Chromium," Department of Enviromental, Food and Rural Affairs, London, Final Report 2005.

[9] D-G. Nam and H-C. Lee, "Thermal nitridation of chromium electroplated AISI316L stainless steel for polymer electrolyte membrane cell bipolar," Journal of Power Sources, vol. 170, n. ${ }^{\circ}$ 2, pp. 268-274, July 2007.

[10] D-H. Han, W.-H. Hong, H. S. Choi, and J. J. Lee, "Inductively coupled plasma nitriding of chromium electroplated AISI 316L stainless steel for PEMFC bipolar plate," International Journal of Hydrogen Energy, vol. 34, n. 5, pp. 2387-2395, March 2009.

[11] P. K. Ajikumar et al., "Surface nitridation of Ti and Cr in ammonia atmosphere," Scripta Materialia, vol. 51, n. 5, pp. 361-366, September 2004.

[12] J. G. Buijnsters, P. Shankar, J. Sietsma, and J. J. ter Meulen, "Gas nitriding of chromium in NH3-H2 atmosphere," Materials Science and Engineering, vol. 341, n. 1-2, pp. 289-295, January 2003.

[13] J. Creus, H. Idrisi, H. Mazille, F. Sanchette, and P. Jacquot, "Improvement of the Corrosion Resistance of CrN Coated Steel by an Interlayer," Surface and Coatings Technology, vol. 107, n. $2-3$, pp. 183-190, September 1998.

[14] J. P. Celis, D. Drees, M. Z. Huq, P.Q. Wu, and M. De Bonte, "Hybrid Processes - A versatile technique to match process requirements," Surface and Coatings Technology, vol. 113, n. ${ }^{\circ}$ 1-2, pp. 165-181, March 1999.

[15] A. Basu et al., "Microstructural and texture studies of gas-nitrided Cr-coated low-alloy high-carbon steel," Surface and Coatings Technology, vol. 201, n.' 16-17, pp. 6985-6992, May 2007.

[16] P. C. King, R. W. Reynoldson, A. Brownrigg, and J. Long, "Ammonia dissociation in the fluidised bed furnace," Materials Forum, vol. 29, pp. 98-102, 2005.

[17] H. O. Pierson, Handbook of Chemical Vapor Deposition: Principles, Technology and Applications, 2nd ed. Albuquerque, New Mexico: Noyes Publications, 1999.

[18] A. Ehrlich, M. Kuhn, F. Richter, and W. Hoyer, "Complex characterisation of vacuum arc-deposited chromium nitride thin films," Surface and Coatings Technology, vol. 76-77, n. ${ }^{\circ}$ Part 1, pp. 280-286, November 1995.

[19] C. Gautier and J. Machet, "Study of the growth mechanisms of chromium nitride films deposited by vacuum ARC evaporation," Thin Solid Films, vol. 295, n. ${ }^{\circ}$ 1-2, pp. 43-52, February 1997.

[20] N. Anacleto and O. Ostrovski, "Solid-state reduction of chromium oxide by methane-containing gas," Metallurgical and Materials Transactions B, vol. 35, n. . 4, pp. 609-615, August 2004. 
